De la fée épique à la fée elfique : Oberon, de Huon de Bordeaux au Midsummer Night's Dream

\title{
Claire Vial
}

\section{(2) OpenEdition}

1 Journals

\section{Édition électronique}

URL : http://journals.openedition.org/shakespeare/941

DOI : 10.4000/shakespeare.941

ISSN : 2271-6424

Éditeur

Société Française Shakespeare

\section{Édition imprimée}

Date de publication : 1 novembre 2002

Pagination : 203-222

Référence électronique

Claire Vial, « De la fée épique à la fée elfique : Oberon, de Huon de Bordeaux au Midsummer Night's Dream », Actes des congrès de la Société française Shakespeare [En ligne], 19 | 2002, mis en ligne le 01 novembre 2007, consulté le 30 avril 2019. URL : http://journals.openedition.org/shakespeare/941 ; DOI : 10.4000/shakespeare.941 


\section{S H A K E S P E A R E \\ \& L E M O Y E N - Â G E}

Société Française Shakespeare

Actes du Congrès de 2001

* * *

Textes réunis et présentés par

Patricia DORVAL

publiés sous la direction de Jean-Marie MAGUIN 


\section{DE L A F E E EPIQUE A L A F EE ELFIQUE : OBERON, DE HUON DE BORDEAUX A U MIDSUMMER NIGHT'S D R E A M}

L'Oberon du Midsummer Night's Dream est l'une des incarnations les plus manifestes de la prégnance du Moyen-Âge littéraire, continental en général et français en particulier, chez les Élisabéthains. À l'origine du personnage de Shakespeare se trouve un petit roi sylvestre, Aubéron, qui est l'un des deux héros de la chanson de geste anonyme Huon de Bordeaux, composée en vers épiques 'entre 1216 et 1268 . Ce premier poème, œuvre à succès, a été l'objet de continuations qui ont porté sa longueur de 10000 à 30000 vers ; l'une de ces continuations en particulier, intitulée le Roman d'Aubéron, s'attache aux origines du personnage tel qu'il apparait dans l'épopée première. Les autres continuations consistent en six poèmes développant les aventures du chevalier Huon et de sa descendance. Le premier poème épique agrémenté de ses continuations fit l'objet au XVe siècle d'un remaniement rimé ; puis, parallèlement, d'un remaniement en prose, vers 1454 , qui reste très proche de l'original. Ce remaniement en prose, dont il ne subsiste aucun manuscrit original, fut édité dans les années 1510 et connut cinq éditions imprimées avant 1550 , ce qui atteste de l'étendue de son succès. Trente-deux éditions en français devaient suivre entre le XIe et le XIXe siècle. C'est vraisemblablement le manuscrit français de 1513, édité par Michel le Noir et conservé à la British Library ${ }^{2}$, qui fut l'objet de l'attention de John Bourchier, Lord Berners, traducteur de 
Froissart, qui en effectua une traduction en 1533 ou 1534. Elle contient, outre le poème original, cinq des continuations : le prologue que constitue le Roman d'Aubéron, la Chanson d'Esclarmonde (épouse de Huon), la Chanson de Clarisse et Florent, la Chanson d'Ide et Olive, la Romance de Croissant. Une édition révisée fut par ailleurs réalisée en 1601, sans doute par le relecteur d'un imprimeur qui en éradiqua les difficultés de langage, sans pour autant défigurer la version de Berners ; seuls quelquesuns des premiers paragraphes furent réécrits dans le style euphuistique en vogue à l'époque ${ }^{3}$.

C'est sans aucun doute la version de Berners de 1534 qui parvint aux yeux de Shakespeare. Elle devait manifester intrinsèquement des qualités dramatiques, puisque l'on retrouve la mention, dans le journal de Henslowe, d'une représentation théâtrale de ce roman d'aventures jouée par les comédiens du Comte de Sussex le 28 décembre 1593 et les 3 et 11 janvier suivants. Shakespeare a fort bien pu connaître cette version adaptée pour la scène, dont il ne reste malheureusement pas de trace manuscrite ${ }^{4}$. Une allusion aux exploits de Huon par Benedick dans Much Ado ${ }^{5}$, confirme la connaissance relativement précise que Shakespeare pouvait avoir de l'épopée ${ }^{6}$.

Dans le poème épique, l'histoire s'ouvre quatre ans après la mort de Séguin, duc de Bordeaux ; ses deux fils, Huon et Gérard, n'ont pas encore rendu l'hommage à l'empereur Charlemagne. Un traître, Amaury, qui convoite l'héritage, les dénonce comme rebelles. L'empereur, âgé et affaibli, décide de les châtier, afin que grâce à cette manifestation d'autorité, tous ses vassaux le reconnaissent au moment même où il songe à abandonner sa couronne à son fils Charlot. Alors que les deux frères sont en chemin vers Paris, le traître Amaury leur tend une embuscade avec la complicité de Charlot ; Gérard est blessé, mais Huon tue Charlot, sans savoir qui il est. Charlemagne jure de se venger de ce meurtre ; bien qu'un duel judiciaire entre Huon et Amaury tourne à l'avantage du bon droit, Charles ne décolère pas et décide de confier à Huon, sous peine de mort, une mission apparemment grotesque et impossible à réaliser. C'est là que commence le voyage aventureux du chevalier vers Babylone; Huon ne réussit à accomplir sa mission et à être réintégré parmi les pairs de France qu'à l'aide du nain Aubéron, roi des fées, dont le royaume se situe quelque part entre Jérusalem et Babylone, et à ses pouvoirs magiques.

On est donc en présence d'une chanson de geste tardive, qui présente un Charlemagne sur le déclin, tout en mêlant des éléments romanesques et des éléments épiques. L'Oberon du Songe se présente comme l'objet d'un triple palimpseste: au premier héros épique se superpose celui du 
remaniement en prose, lui-même traduit par Berners avant d'être réexploité par Shakespeare. Ces trois étapes d'écriture sont aussi trois étapes très marquées dans le temps; elles permettent la mise en évidence du glissement d'un genre, le genre épique, qui perd sa vogue à la Renaissance, et la contamination du genre dramatique par le roman. L'Aubéron primitif et celui de Shakespeare ont cependant en commun, comme une première annonce de leur «compatibilité dramatique», de n'être ni l'un ni l'autre des personnages de papier, mais tous deux tributaires de la parole prononcée. Le poème épique est fait pour être dit et écouté, la pièce pour être jouée, donc dite aussi. Seule l'étape intermédiaire que constitue la prose de Berners se prête à une lecture silencieuse et individuelle. Cette prose porte d'ailleurs des marques caractéristiques d'un remaniement destiné au genre écrit ${ }^{7}$. Le récit est divisé en chapitres, chacun précédé d'une longue rubrique à la mode du XVe siècle, avec l'annonce et le résumé des faits essentiels de chaque division; ces soixante-seize rubriques sont rassemblées en une table précédant le récit dans la prose française. Que reste-t-il du premier Aubéron chez Shakespeare ? Y a-t-il même un terrain commun entre l'enchanteur tout-puissant et l'elfe jaloux ? J'ai tenté d'éclairer quelques aspects de cette métamorphose du merveilleux féerique de la matière épique d'origine en matière comique ${ }^{8}$.

La métamorphose du héros apparaît de la façon la plus manifeste dans les premiers portraits d'Oberon qu'offrent les trois œuvres.

L'Aubéron épique est présenté dans un portrait en pied pour ainsi dire, dans une laisse qui constitue le prologue du poème et où il partage avec Huon le statut de personnage principal de l'épopée. La laisse 1 le mentionne d'ailleurs bien plus longuement que le chevalier ${ }^{9}$. Aubéron est donc un roi sylvestre, à la double ascendance prestigieuse, mêlant un personnage historique mythifié, Jules César, et un personnage légendaire, Morgue. Cette ascendance exclut toute référence au domaine féerique : Morgue sa mère n'y est pas désignée comme une fée. C'est la laisse suivante qui l'indique, sans pour autant insister sur ce détail ${ }^{10}$. Aubéron a donc une double appartenance : chevalerie et féerie, l'univers des châteaux fortifiés et celui de la forêt. Lui-même est fée mais avant tout «un bon chevalier». C'est la coloration chevaleresque qui est privilégiée dans cette première vision du héros. Aubéron synthétise la courtoisie traditionnelle et le merveilleux féerique, les deux étant constamment mêlés dans le genre des chansons de geste, mais plus rarement en un seul personnage. $\mathrm{La}$ dernière notation de ce court prologue annonce «de grands exploits guerriers»; on s'installe donc bien dans le champ référentiel de l'héroïsme. Après ce prologue, on n'entend plus parler d'Aubéron jusqu'à sa rencontre 
avec Huon.

La prose française et anglaise fait abstraction de cette présentation des deux principaux personnages. Le récit débute à la cour de Charlemagne, offrant ainsi une approche plus historique, centrée sur la France et l'empereur". Cette restriction par rapport au poème change notre appréhension du personnage : Aubéron n'est plus un héros a-priori, il est au contraire un antagoniste. Le portrait qui est dressé de lui avant sa rencontre avec Huon est effectué par l'ermite Géraume, un ancien chevalier qui rejoindra les compagnons de Huon pour participer à toutes leurs aventures. La troupe se trouve alors au-delà de Jérusalem, en chemin vers Babylone. Dans la bouche de Géraume, Aubéron devient un ennemi potentiel, le roi des fées habitant une épaisse forêt. Il n'est pas explicitement nain, comme le précise le poème, mais mesure seulement trois pieds de haut. Là encore il est placé sous le signe de l'ambivalence : petit et bossu, il a pourtant un visage angélique («but yet he hathe an aungelyke visage»). Quiconque répond à ses paroles plaisantes est perdu à jamais et ne pourra se défaire de sa présence; celui qui malgré tout s'échapperait de la forêt serait arrêté par le déchaînement des éléments qu'il commande: pluie, vent, grêle et tempête de neige, ainsi que par le surgissement d'une large rivière noire et profonde en travers de son chemin. Mais tout ceci n'est qu'illusion afin de retenir le voyageur auprès d'Aubéron :

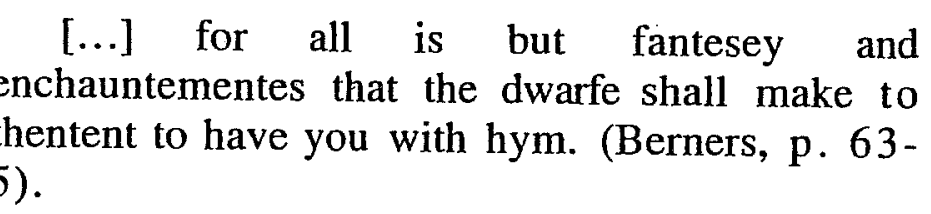

Aubéron semble donc ici un avatar de la figure de la sirène ; il charme et enchaîne par sa parole, sans cependant aller jusqu'à la mort de son prisonnier. On n'a plus, comme dans le poème épique, la juxtaposition d'une vision héroïque et d'une image maléfique, mais seulement cette dernière. L'effet obtenu est celui d'une dramatisation de la rencontre et de la construction du suspense, puisque l'on ne sait pas qu'Aubéron est en fait un bon chevalier.

Avec l'Oberon de Shakespeare, l'héroïsme est entièrement occulté. La première image du roi des fées est fournie à travers le filtre de la parole pas toujours tendre de Puck, qui parle à un elfe de «jealous Oberon», qui va venir se divertir dans les bois ${ }^{12}$. Si l'on a toujours le roi sylvestre, c'est un roi «avec» divertissement, furieux et jaloux depuis longtemps, qui se querelle avec sa reine dès qu'il la rencontre. Fort logiquement, nul portrait 
physique n'en est fait, puisque aussi bien Puck que l'elfe serviteur de Titania connaissent leur roi ; le public a donc un portrait moral partiel d'Oberon pris à un moment donné, mais découvre physiquement le roi lors de son entrée sur scène ; on a là sur le mode plaisant l'équivalent de la rencontre in medias res que fait Huon du roi fée dans la version en prose. Ici, Titania apparaît d'emblée comme le pendant féminin d'Oberon, susceptible de lui résister; elle quitte la scène en paraissant pour un moment avoir le dernier mot ${ }^{13}$. L'Aubéron primitif est dépourvu d'épouse comme de bouffon : le poème épique en matière féminine se concentre peu, avant la fin du XIIe siècle, sur l'élément féminin, sauf à ce qu'il fasse réellement partie des aventures, ce qui est le cas ultérieurement dans Huon avec le personnage d'Esclarmonde.

Non seulement les personnages, mais aussi les valeurs qu'ils incarnent, semblent très éloignées les unes des autres, malgré l'identité de statut des deux Au/Oberon.

L'Aubéron de l'épopée comme celui du roman sont des rois craints, tout-puissants, qui règnent effectivement sur des vassaux. Il suffit de considérer les appellatifs qui les concernent pour mesurer à quel point ils appartiennent à la sphère de la courtoisie chevaleresque, bien qu'étant fées. Lors de sa première rencontre avec Huon, le roi fée est accompagné de quatre cents chevaliers fées ; ils s'adressent à lui en employant les termes courtois de «noble seigneur»" ${ }^{14}$, «syr» dans la prose; quand Huon se soumet au pouvoir d'Aubéron il utilise lui aussi le mode d'expression vassalique : «Seigneur, je ne discuterai pas votre volonté» ${ }^{15}$; en anglais : «I wyll folowe your pleasour, and neuer do nor thynke the contrary» ${ }^{16}$. Les appellatifs de l'Aubéron épique oscillent, au gré des humeurs de Huon et de son ressentiment pour le roi fée quand celui-ci lui refuse son aide, entre l'ironie et le respect: Géraume parle alors du «nain bossu», en anglais «the crokyd dwarfe of ye Fairey», du «nain rusé et opiniâtre», voire du «démon» («devyll») ${ }^{17}$; Huon ajoute l'épithète de «nain bossu et puant»", imité plus tard par Charlemagne ${ }^{19}$. Ces termes vigoureux impriment un décalage cocasse avec le ton plus mesuré du conteur, qui parle du «vaillant Aubéron» ${ }_{22}^{20}$, qui est aussi «vertueux», «noble»" ${ }^{21}$, «valeureux» ou «plein de sagesse» ${ }^{22}$ et souvent tout simplement «le roi Aubéron». Celui-ci connaît et applique parfaitement les règles de courtoisie: «a', quod Oberon, thy salutasyon shalbe well rewardyd ${ }^{24} ;$ «Huon, cher ami, ton salut a été courtois» ${ }^{25}$. Le poème et la prose le présentent en majesté, dans son palais, sur son trône magique; dans le poème, il respecte les lois de l'hospitalité seigneuriale en taillant lui-même devant Huon pain et 
nourriture ${ }^{26}$. Il sait aussi manifester pitié et générosité envers les païens vaincus qui acceptent de se convertir ${ }^{27}$. Enfin, s'il lui arrive d'être vindicatif, ses colères sont toujours déclenchées par les transgressions de Huon, à qui il demeure pourtant fidèle et porte secours régulièrement.

Le roi des fées de Shakespeare, en fait d'épithète homérique, est affublé dans ses premières apparitions du qualificatif «jealous»: il s'adresse à Titania lors de leur première entrevue en l'appelant «proud Titania» ou «rash wanton» ${ }^{28}$; elle le lui rend en reprenant le «jealous Oberon» de Puck ou en lui reprochant «his brawls». En fait d'autorité royale, c'est elle qui, en refusant de se plier au souhait qu'il a exprimé qu'elle lui donne son page, incarne la rébellion et génère le dysfonctionnement chez les fées. C'est là le premier ressort, presque vaudevillesque, de la comédie ; une situation impensable dans l'épopée. Titania mène pour partie une existence indépendante, avec ses prêtresses, ses pages et ses serviteurs qui lui obéissent parfaitement. Les fées minuscules sont toujours prêtes («ready !») à la servir avec application ${ }^{29}$, s'inclinent devant la reine et Bottom et connaissent les règles de courtoisie : «be kind and courteous to this gentleman [...] / Nod to him, elves, and do him courtesies» commande la reine ${ }^{30}$. Elles obéissent immédiatement aux demandes un peu familières de Bottom, et le servent avec une politesse qui confine à la timidité : «Pray you, leave your curtsy, good monsieur», commande Bottom à Mustardseed avec bienveillance ${ }^{31}$; mais leur courtoisie est aérienne et non pesamment chevaleresque. Si Oberon est le maître de Puck, ce rapport ne s'exprime pas sous la forme codifiée du langage vassalique, mais par de seuls impératifs («Fetch me this herb», II.1.173). Les appellatifs utilisés par Puck, contrairement au poème épique, rappellent systématiquement la qualité féerique du roi : «Captain of our fairy band», «King of Shadows» ou «my fairy lord» ${ }^{32}$. Par contraste, la fonction royale d'Oberon et la subordination de Puck sont donc moins soulignées. À aucun moment le lien qui les unit n'est exprimé sous la forme de la soumission ou d'un engagement réciproque; la seule exception à cette pratique, quand Puck souligne au contraire son obéissance à Oberon, sonne davantage comme une parodie de serment d'obéissance de Puck qui joue à faire du zèle : «Fear not, my lord, your servant shall do so» ${ }^{33}$.

L'opposition entre le ton sérieux, associé à l'épopée et au roman et le ton comique, associé à la pièce, se retrouve en apparence lorsque l'on considère le type d'intervention des rois-fées dans les affaires humaines. L'Aubéron épique est accompagné de chevaliers fées qui font office de guerriers à l'occasion; il est en lien avec les institutions de la papauté et 
de l'Empire. Ses interventions visent d'une part à combattre les Infidèles ; ce qu'il fait grâce à ses troupes mobilisables instantanément de cent mille hommes en armes qui «emplissent les rues» ${ }^{34}$; à plusieurs reprises, il promet la vie sauve aux païens qui acceptent de recevoir le baptême ${ }^{35}$. Fidèle à son serment, il porte secours à Huon dans la cité sarrasine de Tormont $^{36}$, et à Babylone ${ }^{37}$, bien que le chevalier se soit montré peu fiable dans son obéissance à ses commandements. D'autre part, il rétablit Huon dans ses droits en allant avec ses troupes jusqu'à Bordeaux, où il fait obstacle aux troupes de Charlemagne. Enfin, il tient même les fonctions de juge suprême et de procureur, en quelque sorte ; il dévoile la mauvaise foi de Charlemagne qui refuse de reconnaître l'innocence de Huon ${ }^{38}$, oblige Gérard, le frère de Huon, à avouer sa traîtrise et prononce une sentence de mort pour les traitres, que ses pouvoirs magiques lui permettent d'appliquer instantanément $^{39}$ : il lui suffit de les souhaiter au gibet pour qu'ils s'y retrouvent pendus. L'éthique de l'épopée s'applique donc à faire respecter une justice chrétienne et féodale, fondée au plan individuel sur l'allégeance et le respect de la prescription.

À côté de ces interventions qui se situent au niveau des institutions, l'Oberon de Shakespeare ne semble interférer avec les affaires athéniennes que sur le mode apparemment ludique ou frivole. La raison invoquée par Titania à la première scène de l'acte II pour expliquer la présence d'Oberon à Athènes est d'assurer le bonheur et la prospérité au couple de Thésée et d'Hyppolite $^{40}$. Quant aux troubles qui perturbent son propre royaume troubles intérieurs totalement absents du royaume féerique de l'épopée où Aubéron fait régner la justice seigneuriale - ils s'enracinent dans la jalousie et se résolvent par une revanche obtenue par la ruse et l'enchantement. Ce qui n'empêche pas Oberon d'être authentiquement généreux puisqu'il décide également, en déléguant une partie de ses pouvoirs à Puck, de réinstaurer l'équilibre amoureux entre Démétrius et Héléna, d'abord ${ }^{41}$, puis entre les quatre jeunes gens perdus dans les bois ensuite, pour réparer l'erreur de Puck. La justice à demi intéressée dispensée par Oberon est circonscrite au cercle des relations humaines et donc, en apparence en tout cas, superflue du point de vue du fonctionnement des institutions. L'Aubéron primitif défenseur du «bon droit», s'il était transposé dans la pièce de Shakespeare, serait du côté d'Egeus, le père de Hermia, voire de Thésée, qui applique la loi mais accepte de l'assouplir temporairement ${ }^{42}$.

Pourtant, l'impact des conflits et de leur résolution atteint chez Shakespeare des dimensions cosmiques que n'expriment jamais les résolutions politiques de l'épopée. Celle-ci s'enracine dans une forme de 
réalisme historique, ou de prétention au réalisme ; au cœur du conflit se trouve un empereur vieillissant dont le jugement n'est plus équitable, des traîtres, des pairs du royaume incapables de s'unir autour d'un jugement unanime. Si l'intervention féerique résout dans l'épopée première des difficultés que les seuls humains ne parviennent pas à maîtriser, la version en prose de Berners - suivant l'original français - indique que seule une résolution temporaire des conflits a été atteinte. Ce dénouement partiel est bénéfique au plan narratif, puisque c'est ainsi que se prépare la continuation des aventures, dont l'ultime et heureuse conclusion ne sera que très indirectement imputable à Aubéron. Il n'en reste pas moins que le retour total à la stabilité, rendu possible par l'intervention du merveilleux chrétien dans le poème épique, est immédiatement remis en cause dans la version romanesque. Ce qui est un autre trait caractéristique du remaniement continu des chansons de geste entre les XIIe-XIIIe siècles et le $\mathrm{XVe}$ siècle, remaniement qui s'effectue au profit de rebondissements aventureux qui paraissent sans fin. On pourrait être tenté d'y voir aussi les traces d'une interrogation quasi-inconsciente du fonctionnement des institutions contemporaines du remaniement ${ }^{43}$.

Chez Shakespeare, quoi qu'il en soit, Titania rappelle en II.1.88-117, le désordre dans les éléments naturels engendré par la querelle des époux féeriques ; dans l'économie de la pièce, ce désordre fait aussi écho aux discordances familiales et amoureuses chez les humains. Quant à Oberon, après avoir convaincu Titania de lui donner le page qu'il convoitait et l'avoir délivrée de son propre enchantement, il se proclame restaurateur de l'harmonie universelle (III.2.377 : «and all thing shall be peace»). Après avoir endormi les Athéniens dans le bois, le roi et la reine des fées enfin réunis dansent auprès d'eux ; c'est à nouveau Oberon qui constate le retour à l'harmonie entre les couples et garantit des augures favorables pour les mariages du lendemain (IV.1.83-8). La ronde finale des fées de pièce en pièce dans le palais ducal matérialise ce retour à l'harmonie universelle.

Ainsi, le roi chevalier de Berners et le roi jaloux de Shakespeare semblent n'avoir guère en commun que leur nom et leur royaume en Inde ${ }^{44}$. On ne trouve plus trace des valeurs épiques chez Shakespeare, où elles ne sont plus au goût du jour ; la royauté d'Oberon semble davantage une parodie de l'autorité déployée par le roi fée du poème primitif. Reste à examiner la facette proprement féerique du personnage.

Le roi-fée primitif est un bon exemple du syncrétisme de diverses traditions littéraires. Le poème épique en fait un intermédiaire privilégié entre la féerie et le monde chrétien. Il n'en est pas de même pour les fées shakespeariennes. 
Le prologue du Huon épique présente en effet un personnage rassemblant la matière de Rome et la matière de Bretagne. Il est le fils de Jules César et de Morgue, «une dame de grande sagesse» d'après la laisse. 1, qui ne mentionne pas la nature féerique de Morgue. Cette alliance de deux matières littéraires distinctes est la première d'une série de paradoxes qu'Aubéron rassemble en sa personne. À Huon il apparaît comme un enfant (la prose de Berners indique simplement qu'il est petit trois pieds de haut ${ }^{45}$ ) et un homme mûr, né avant Jésus-Christ. Il est nain et bossu, mais en même temps selon le poème, d'une beauté solaire : «sa beauté éclipse celle du soleil en été» ${ }^{\text {t6 }}$, décrit l'ermite Géraume. Le conteur reprend l'expression à son compte lorsqu'il cite la première rencontre entre Aubéron et la troupe de Huon : «il est aussi beau que le soleil d'été» ${ }^{47}$ ou «au visage angélique» dans la version anglaise. Ce rayonnement est repris par celui de son habit, «fait d'une pièce de soie striée, avec trente bandes d'or pur, que des fils de soie retiennent sur les côtés»; dans la prose, sa tenue est garnie de pierres précieuses qui brillent comme le soleil ${ }^{48}$. Cette beauté solaire et angélique ne l'empêche pas de se faire traiter de «démon» par Huon et Géraume quand ils prennent conscience qu'ils ne pourront plus lui échapper ${ }^{49}$. C'est alors qu'Aubéron réplique en explicitant sa nature : «for I was neuer devyll nor yll creature / I am a man as other be. But I coniure the by the deuyne puisance to speke to me» ; «par celui qui fut pendu sur la croix, je ne suis ni un démon ni une créature diabolique, mais, sur mon salut, je t'affirme que je suis un homme comme les autres. Je viens donc vous adjurer au nom de Dieu, par tout ce qu'il a créé et façonné, par l'huile, le chrême, le sel et le baptême, et par le pouvoir que Jésus m'a remis, de répondre à mon salut» ${ }^{50}$. C'est là que réside son syncrétisme original, réaffirmé sans cesse dans le poème quand Aubéron clame qu'il agit en vertu «du pouvoir prodigieux que Jésus [lui] a donné, en Féerie, [son] domaine»". Son merveilleux féerique est donc bien de type chrétien, cependant que son visage angélique et certains de ses attributs magiques, comme son hanap, portent la marque du merveilleux celtique ${ }^{52}$. Son domicile «en Inde», au-delà de Jérusalem, lui apporte en outre une coloration orientalisante.

Les récits français et anglais en prose étoffent la généalogie d'Aubéron : sa mère n'est plus Morgue, mais seulement une dame exilée dans «l'Isle celée» («the lady of the pryvey Isle») nommée «Chifalonnye» (Céphalonie). Le merveilleux païen ne se mélange donc pas, dans la prose, au merveilleux chrétien; en revanche, la mère d'Aubéron y gagne «une aura romanesque et mystérieuse» ${ }^{54}$. Lorsqu'il 
s'adresse pour la seconde fois à Huon, Aubéron répète l'histoire de ses origines en spécifiant dans le poème les dons que les fées lui ont conférés à sa naissance : l'omniscience, le pouvoir de se transporter instantanément et avec qui il souhaite en tout pays, et celui de faire apparaître toutes sortes de constructions tel château ou navire ${ }^{\text {ss }}$. Il peut soumettre les animaux sauvages et connaît (seulement dans le poème) tous les secrets de paradis où une place lui est réservée auprès de Dieu ${ }^{56}$. On a vu auparavant qu'il peut commander aux éléments naturels et faire surgir toutes sortes d'illusions.

La plupart de ces spécifications sont absentes dans l'univers plus éphémère et moins construit du mari de Titania. En matière de science, il connaît le pouvoir des fleurs et leur influence bénéfique ou maléfique ${ }^{57}$; il sait se rendre invisible ${ }^{58}$ et connait les formules d'enchantement ${ }^{s 9}$. Il sait faire surgir la musique qui endort les humains ${ }^{60}$. Comme l'Aubéron primitif, il peut commander aux éléments et demande ainsi à Puck d'assombrir la nuit par des brouillards ${ }^{61}$. Il est donc plutôt un enchanteur, connaisseur de sortilèges et en aucun cas un envoyé divin. Tout comme Puck, Titania et ses minuscules serviteurs, il est susceptible d'apparaître et de disparaître instantanément et impalpablement. Il peut voir les humains mais la réciproque n'est pas vraie. Bien que Keith Thomas rapporte la croyance à l'époque élisabéthaine en un petit peuple des fées, nain et espiègle, mais bienveillant ${ }^{62}$, rien ne permet de dire dans la pièce qu'Oberon et Titania ont une taille inférieure à celle des humains; la tradition scénique a d'ailleurs souvent recours aux mêmes acteurs pour interpréter les deux couples royaux de la cité et de la forêt ${ }^{63}$. Même si Oberon indique que les fées de son espèce ne craignent pas la lumière du jour ${ }^{\circ 4}$, un cloisonnement strict s'instaure entre l'heure des fées, «fairy time», mentionnée par Thésée, et l'heure des mortels qui commence avec le chant de l'alouette ${ }^{65}$. On est loin du merveilleux romanesque qui se manifeste au cour des aventures humaines et dont les prodiges sont intégrés au quotidien. La nature même de la fée est exprimée de façon divergente dans les œuvres: le terme «fée» ou «fairy» est le seul à désigner Aubéron dans le poème et la version en prose, mis à part le titre injurieux de «démon» déjà cité, qui lui est attribué dans un accès de courroux pour être démenti juste après. La nature profonde d'Obéron et de son peuple est bien plus diffuse et difficile à cerner : il est le «roi des ombres»" appartient au «peuple des esprits»" Titania est suffisamment bien en chair pour faire sa cour à Bottom, mais elle promet de le rendre semblable à elle, «a spirit of no common rate» et aussi «an airy spirit» ${ }^{68}$. 
Cette imprécision délibérée n'empêche pourtant pas un renversement curieux : si l'Aubéron primitif est explicitement un «petit roi sylvestre» qui tente de piéger les voyageurs au cœur de la forêt, c'est bien chez Shakespeare que la magie nocturne des bois près d'Athènes est la plus prégnante et la plus palpable. C'est là que la forêt se manifeste comme un autre monde, source de renversement, d'aveuglement ou de révélation, bien plus que dans la forêt typique, aux mystères attendus, de l'épopée ou du roman.

L'exploration de la nature du merveilleux dans les œuvres n'est complète que si l'on examine les êtres féeriques avec lesquels nos différents $\mathrm{Au} /$ Oberon interfèrent.

L'Aubéron épique règne sur une grande quantité de chevaliers fées ${ }^{69}$. Des auxiliaires qui peuvent s'armer pour lui obéir, ce qu'ils font sans discuter. Son autorité n'exclut pas pourtant la possibilité d'être interpellé et contredit, dans les limites de l'étiquette courtoise : c'est ce que fait le chevalier Gloriant, un chevalier fée qui demande par deux fois à Aubéron de faire preuve de tolérance et de grandeur d'âme envers un Huon qui ne cesse de transgresser ses interdits ${ }^{70}$. Aubéron a aussi quelques serviteurs à qui il délègue une partie de son pouvoir : un messager fée dont la baguette d'or sépare les rivières et permet aux humains de les traverser". Et surtout le génie marin Malabron qui aide Huon à traverser la mer Rouge dans le poème ${ }^{72}$ et aussi le Nil, au milieu des crocodiles et des serpents, dans la version de Berners. Comme son maître, Malabron est capable de prévoir l'avenir et les malheurs qui vont s'abattre sur Huon ; il est à l'origine un homme lige d'Aubéron à qui il a désobéi et a été condamné à être pendant trente ans un animal marin ${ }^{73}$. Dans la version en prose, Huon en le rencontrant, commence par se signer et tirer son épée, puis lui demande s'il est du côté de Dieu ou des mauvais esprits. Sa soumission à la volonté d'Aubéron et sa bienveillance pour Huon le mènent jusqu'au sacrifice de sa personne, puisqu'il propose d'aider le chevalier après une nouvelle désobéissance de ce dernier en acceptant en contre-partie un renouvellement de sa peine pour vingt-huit ans dans le poème, dix chez Berners ${ }^{74}$.

Aubéron et ses émissaires ne sont pas les seuls représentants du merveilleux : dans le poème original comme dans ses continuations, le chevalier est confronté à un démon, à des anges déchus déguisés en moines dotés de pouvoirs magiques; à un géant dont l'armure rend invulnérable et à un gryphon. On retrouve ici des éléments traditionnels du merveilleux chrétien et des récits de Mandeville. Ces obstacles sont surmontés par Huon grâce à sa force, sa ruse, son courage et parfois l'aide d'objets 
magiques fournis par Aubéron. Du point de vue de ce dernier, l'effet de mimétisme avec le modèle chevaleresque le place vis-à-vis de ses auxiliaires féeriques dans une position de supériorité identique à celle du seigneur envers ses chevaliers. Par son autorité et surtout grâce à son cor magique qui lui permet de les convoquer instantanément pour leur attribuer une tâche, Aubéron se trouve une fois de plus dans la position intermédiaire de l'être, certes fée, mais qui, en tant que seigneur, soumet les autres personnages féeriques ; tout ceci est toujours effectué «par magie et par la grâce de Dieu», en prose «by the wyll of god and the power that he had in the fayrye» ${ }^{75}$ : on a donc affaire à une exploitation des forces féeriques au profit du bon droit chrétien ${ }^{76}$.

Oberon se trouve dans une tout autre position ; il dispose d'un seul maître d'œuvre, féerique ou qui se définit comme tel, Puck : «we faieries, that do run by the triple Hecate's team...»" . Puck est à la fois le bouffon d'Oberon et son héraut ( $« \mathrm{I}$ jest to Oberon, and make him smile», II.1.44); mais dans le dialogue avec le serviteur fée de Titania où il indique ce rôle de bouffon, l'on perçoit qu'il est largement autonome dans ses espiègleries. C'est un esprit vagabond («wanderen»), qui fait son quotidien des tours qu'il joue aux villageoises et aux voyageurs ${ }^{78}$. Il peut se transformer en animal, en végétal ou en objet animé ${ }^{79}$ et imiter les voix humaines. Si dans Huon de Bordeaux, le génie Malabron se permet par générosité d'intercéder pour Huon en payant de sa personne par un châtiment augmenté, Puck a clairement une existence féerique en dehors des services qu'il accomplit pour Oberon, et ne les accomplit pas toujours avec exactitude; sa méprise s'avère comme on sait essentielle aux péripéties dramatiques. C'est lui qui prend l'initiative de parachever la vengeance d'Oberon en revêtant Bottom d'une tête d'âne ${ }^{80}$; il s'amuse à cette occasion à poursuivre les artisans en répétition, en précisant l'étendue de sa propre magie transformatrice ${ }^{81}$; on en apprend donc bien plus sur ses propres pouvoirs que sur ceux de son roi. Quant aux fées minuscules de Titania, ce sont des fées et des champs et des forêts qui ont des tâches très précises et sérieuses à effectuer sur le chemin de leur reine ${ }^{82}$, pour la distraire de leurs chants et de leurs danses, ou s'occuper des fleurs, des insectes et animaux des bois ${ }^{83}$.

La nature de nos Obéron tout comme les fonctions qui leurs sont attribuées dans leurs œuvres respectives tendent à confirmer l'absence de terrain commun entre une fée épique d'un côté et une fée elfique d'un autre. 
Il est pourtant un domaine, qui tient paradoxalement à la nature spécifique des œuvres dans lesquelles ils apparaissent, qui les fait se rejoindre. Le personnage du poème, dont les exploits sont faits pour être dits et celui de la pièce, qui ne peut que dire et faire devant les spectateurs, sont tous deux détenteurs d'une parole qui façonne les faits et gestes des protagonistes, d'une parole contraignante.

Le premier sortilège qui unit de toute éternité Aubéron et ses victimes passe par la parole: nul ne peut lui parler sans rester avec lui pour toujours; quiconque traverse son territoire est obligé de lui parler ${ }^{84}$. En vérité, c'est l'ensemble du poème épique qui fonctionne sur le mode de la parole donnée, puis respectée ou retirée. Les deux frères, fils du duc de Bordeaux, sont d'abord coupables auprès de Charlemagne de n'être pas venus lui rendre hommage après la mort de leur père ; le traître Amaury, pour s'emparer de leur territoire, répand le bruit de leur rébellion; enfin Huon n'a la vie sauve que parce qu'il fait le serment à Charlemagne d'accomplir une série d'exploits. Lors de la première rencontre, Aubéron commence par saluer les chevaliers «au nom du Dieu de majesté» en les adjurant de répondre à son salut ${ }^{85}$; les chevaliers fuient une première fois, bien que Huon soit attiré par cet homme curieux, qui est d'une beauté parfaite et qui «parle bien de Dieu» ${ }^{86}$; ils finissent par être rattrapés par le nain qui à nouveau leur adresse la parole et propose son aide à Huon (dont il connait parfaitement la mission) : en somme, ses pouvoirs magiques en échange d'une salutation. Huon est dévoré de curiosité et finit par rendre le salut. Sa soumission quasi-vassalique à Aubéron ensuite n'est exprimée que par une parole d'engagement: «Seigneur, je ne discuterai pas votre volonté» ${ }^{87}$; chez Berners : «syr, I wyll folowe your pleasour, and neuer do nor thynke the contrary» ${ }^{88}$. Une fois cette alliance scellée et les chevaliers transportés dans le palais de Huon, le roi-fée décide d'aider le chevalier dont il admire la droiture en lui donnant des objets magiques : un hanap et un cor d'ivoire. Tous deux sont soumis à un interdit verbal ou assimilé. Le hanap procure du vin à satiété à tout homme vertueux, «mais si tôt que tu proféreras un mensonge, le pouvoir du hanap t'échappera et tu perdras complètement mon amitié». De même avec le cor d'ivoire, qui permet à celui qui souffle dedans d'appeler Aubéron où qu'il se trouve et de le convoquer avec cent mille hommes d'armes, à condition de ne pas en sonner en vain, c'est-à-dire de ne pas mentir sur la nécessité où l'on se trouve de demander de l'aide ${ }^{89}$. Enfin le génie marin Malabron, après avoir fait traverser la mer Rouge à Huon, lui donne un seul avertissement de la part de son maître pour survivre aux vicissitudes qui l'attendent dans la 
ville de Babylone: «dès que tu proféreras un mensonge, tu perdras l'amitié d'Aubéron»" ${ }^{\circ}$. Quand Huon sonne du cor pour demander de l'aide à Aubéron, c'est sur un simple souhait que celui-ci se trouve transporté avec son armée à Babylone : «Il s'y trouve aussitôt qu'il l'a dit ; c'est œuvre de magie, et c'est par la volonté de Dieu»". Il se transportera plus tard de la même manière au palais de Charlemagne, pour établir l'innocence de Huon. C'est à nouveau par la parole qu'Aubéron met à jour la fausseté de l'empereur et la traîtrise de Gérard, le frère de Huon ; c'est aussi sur la foi de son récit des aventures de Huon que l'honneur de ce dernier est restauré $^{92}$. On a donc affaire à une parole d'Aubéron contraignante et performative. Le dire est pour lui le moyen premier du faire, qu'il s'agisse de ses propres désirs ou de la coercition qu'il peut exercer sur les autres.

La magie d'Oberon chez Shakespeare s'exprime dans des interventions moins variées, mais le moyen est le même. Oberon agit soit en donnant des ordres à Puck «fetch me that flower... fetch me this herb» ${ }^{93}$; soit en prononçant des enchantements au-dessus de Titania et Demetrius. Ces charmes sont constitués de tétramètres rimés particulièrement riches en assonances ${ }^{94}$; de même le chant final qu'il entonne et qui est repris par les fées a une double fonction, protectrice du point de vue de l'intrigue et conclusive du point de vue dramatique.

Finalement, si l'on veut cemer l'évolution de la nature littéraire des fées dans ces œuvres, la coloration globale de chacune est la suivante. Comme toujours chez les médiévaux, le merveilleux est considéré largement comme allant de soi. Ainsi, Aubéron est fée, sans ambiguïté. Nous connaissons sa personne, ses humeurs et ses pouvoirs; son statut de fée n'est jamais remis en question. Ses capacités, tout aussi extraordinaires qu'elles soient, ne surprennent que ceux qui ne connaissent pas le personnage et n'ont plus rien d'imprévisible pour les autres. De même, dans l'épopée, les objets magiques sont pluriels et décrits dans leur aspect comme dans leurs fonctions; ils sont absents chez Shakespeare. Le petit roi fée épique fonctionne suivant des catégories morales chevaleresques qui sont d'autant plus facilement identifiables que le récit les rappelle régulièrement: le respect de la prescription, de la parole donnée, la générosité : autant de vertus qui sont chevaleresques avant d'être féeriques. Au contraire, les fées shakespeariennes du Songe tirent l'essentiel de leur charme du fait qu'elles demeurent insaisissables, quand bien même le couple royal renvoie à celui de Thésée et Hyppolyte dans le monde diurne 
et bien ordonné des humains : «we shadows», rappelle Puck dans son dernier chant... Nulle indication scénique ne vient préciser leur physique ou leur accoutrement ; on sait simplement que les serviteurs elfes de Titania sont minuscules, vu leurs noms et le genre de cachette qu'ils choisissent ${ }^{96}$. Les bois près d'Athènes ne sont que leur domaine provisoire, puisque Oberon est censé venir de son royaume en Inde. Mis à part les serviteurs obéissants de la reine, les fées de la pièce sont également imprévisibles dans leurs erreurs, leur obstination ou leur générosité : par contraste avec la fée épique ou romanesque, la magie féerique du Songe est une magie de l'indéfinition. On a donc dans l'épopée un merveilleux très divers mais très codifié, qui suscite la curiosité de l'auditoire mais non son interrogation quant à la nature des merveilles évoquées devant lui ${ }^{97}$. Au contraire dans le Songe, à l'instar des Athéniens qui ne peuvent ni voir les fées ni garder souvenir de leur intervention, pourtant déterminante dans les affaires humaines, le spectateur ne sait pas, exception faite de Puck, à quel type de fée il a affaire : même l'étiquette «fairy» n'est pas stable.

On ne peut achever ce parcours sans évoquer brièvement les autres Oberon, contemporains de Shakespeare. Celui de Greene, dans The Scottish Historie of James the Fourth, Killed at Flodden (1598, mais jouée sans doute vers 1590$)^{98}$, ne se manifeste qu'entre les actes pour mener des «antiques» et faire surgir devant son ami écossais Bohan des images exotiques de souverains légendaires comme Sémiramis ou Cyrus. Il apparaît essentiellement comme un maître des plaisirs et des illusions, offrant ainsi un contrepoint divertissant au drame historique ; il accorde en outre aux deux fils de Bohan, dont l'un est nain, comme lui, des dons susceptibles de leur procurer une existence prospère et variée ${ }^{99}$.

Reste le masque de Ben Jonson, Oberon the Fairy Prince, a masque of Prince Henry's, joué en $1611^{\text {ioo }}$. Oberon-Henry y apparaît servi par les grands chevaliers de jadis qui reçoivent dans le palais féerique une seconde vie et une jeunesse éternelle. Ils se sont rassemblés pour rendre leur hommage annuel au roi Arthur. Ultime retour des choses : on retrouve en cet Oberon-là le héros solaire et chevaleresque du poème épique, «lovelier than in May is the spring». Fils âné de Jacques Ier, le prince incarne bien sûr la vertu et la bienveillance du bon monarque, tout en maintenant son royaume dans une éternelle jeunesse :

'Tis he that stays the time from turning old,

And keeps the age up in a head of gold

That in his own true circle still doth run; 
And holds his course as certain as the sun.

He makes it ever day, and ever spring, Where he doth shine, and quickens everything, Like a new nature...

Claire VIAL Université de Paris III - Sorbonne Nouvelle

\section{N O T E S}

'Laisses de vers décasyllabiques assonancés avec césure régulière après la quatrième syllabe.

${ }^{2}$ Michel J. Raby, Le Huon de Bordeaux en prose du XVe siècle, NewYork, Washington, Paris, Peter Lang, 1998 ; désormais abrégé en Raby. Ici Introduction, chapitre I: Tradition manuscrite et imprimée de Huon de Bordeaux et de ses continuations.

${ }^{3}$ S. L. Lee, The Boke of Duke Huon of Burdeux done into English by Sir John Bourchier, Lord Berners, and printed by Wynkyn de Worde about 1534 AD, Early English Texts Society, Extra Series, 40, 41, 43, 50, London, 18821883 ; désormais abrégé en Berners. Les citations du texte renvoient toutes, sauf exception signalée, au volume 40 , les volumes suivants étant consacrés à la traduction des continuations. Ici vol. 50, Introduction.

${ }^{4}$ Berners, vol. 40, p. xlix.

III.1.263.

${ }^{6}$ Berners, vol. 40, p. li.

'Raby, Introduction, chapitre I.

${ }^{8} \mathrm{Je}$ remercie tout particulièrement Margaret Jones-Davies et Pierre Kapitaniak pour leurs précieuses suggestions bibliographiques concernant les figures d'Oberon contemporaines de celle de Shakespeare.

Les citations se rapportent à l'édition en français moderne effectuée par François Suard, Histoire de Huon de Bordeaux et Aubéron, roi de féerie, Paris, Stock, 1983 ; désormais abrégé en Huon. Ici, citation p. 19.

Huon, p. 20. 
"Berners, p. 63-4; voir aussi Raby, Introduction, p. cxi.

12

II.1.18-31.

II II.1.145.

${ }^{14}$ Laisse 26, entre autres.

${ }^{15}$ Laisses 27, 36, 64, 91.

${ }^{16}$ Berners, p. 74.

${ }^{17}$ Huon, laisses 25 et 26 ; Berners, p. 68.

${ }^{18}$ Laisses 61, 68, 72.

${ }^{19}$ Laisse 91.

${ }^{20}$ Laisses 26, 38.

${ }^{21}$ Laisse 69.

${ }^{22}$ Laisse 38 .

${ }^{23}$ Laisses 1, 37, 81, 85, 91.

${ }^{24}$ Berners, p. 72.

${ }^{25}$ Laisse 26.

${ }^{26}$ Laisse 30.

${ }^{27}$ Laisse 36 ; Berners, p. 95.

${ }^{28}$ II. 1.60-145.

${ }^{29}$ III. 1.143-76.

${ }^{30}$ III.1.145 \& 155.

${ }^{31}$ IV.1.17-8.

${ }^{32}$ Respectivement III.2.110 \& 347.

${ }^{33}$ II. 1.268.

${ }^{34}$ Huon, laisse 66 ; Berners, p. 152.

${ }^{35}$ Huon, laisse 66 ; Berners, p. 152-3.

${ }^{36}$ Huon, laisse 36 ; Berners, p. 94-5.

${ }^{37}$ Berners, p. 152.

${ }^{38}$ Huon, laisse 91 ; Berners, p. 261.

${ }^{39}$ Huon, laisse 91 ; Berners, p. 264-5.

${ }^{40}$ II. 1.72-3.

${ }^{41}$ II. 1.244-67.

${ }^{42}$ I. 1.83-90.

${ }^{43}$ Voir Robert Bossuat et alii, Dictionnaire des lettres françaises, le 
Moyen-Âge, Paris, Fayard, 1994 (1964), article «chanson de geste».

II.1.22, $69 \& 124$.

${ }^{45}$ Berners, p. 63.

Huon, laisse 24.

${ }^{47}$ Laisses $24 \& 32$.

${ }^{48}$ Huon, laisse 24 ; Berners, p. 65.

${ }^{49}$ Laisse 26.

so

Huon, laisse 26 ; Berners, p. 69.

${ }^{51}$ Laisse 30.

52

Berners, vol. 50, p. xxxii.

53

Berners, p. 72.

${ }^{54}$ Raby, Introduction, chapitre 4, p. cxi sq.

${ }^{55}$ Laisse 26.

${ }^{56}$ Laisse 28.

${ }^{57}$ II. 1. 148-85.

II. 1. 186-7.

II $2.35-42$.

${ }^{60}$ IV . 1.42.

${ }^{61}$ III. 2.355-6.

${ }^{62}$ Keith Thomas; Religion and the Decline of Magic, London, Penguin Books, 1982 (1971), p. 726.

${ }^{63}$ Je remercie le professeur François Laroque pour cette précision.

${ }^{64}$ III.2.388.

${ }^{65}$ IV. 1.98

${ }^{66}$ III.2.147.

III. 2.388.

${ }^{68}$ III. $1.135 \& 142$.

${ }^{69}$ Huon, laisse 26 ; Berners, p. 70.

${ }^{70}$ Huon, laisses 58 \& 102 ; Berners, p. 70.

"Huon, laisse 30 ; Berners, p. 78.

${ }^{72}$ Laisse 44.

${ }^{73}$ Huon, laisse 45 ; Berners, p. 111.

${ }^{74}$ Huon, laisse 68 ; Berners, p. 112. 
${ }^{75}$ Berners, p. 153 ; les exemples sont multiples.

${ }^{76}$ Voir aussi Pierre-Yves Badel, Introduction à la vie littéraire du MoyenÂge, Paris, Bordas, 1969, p. 129.

${ }^{77} \mathrm{~V} .1 .365$.

${ }^{78}$ II. $1.32-42$.

${ }^{79}$ II. $1.42-58$.

${ }^{80}$ III. 2.16-7.

${ }^{81}$ III.1.94-9.

${ }^{82}$ II. $1.1-17$.

${ }^{83}$ II. 2.1-34.

${ }^{84}$ Huon, laisse 24 ; Berners, p. 63.

${ }^{85}$ Huon, laisse 24 ; Berners, p. 69.

${ }^{86}$ Huon, laisse 26 ; Berners, p. 71.

${ }^{87}$ Laisse 27.

${ }^{88}$ Berners, p. 74.

${ }^{89}$ Huon, laisse 30 ; Berners, p. 77-8.

${ }^{90}$ Huon, laisse 46, c'est moi qui souligne ; Berners, p. 113.

${ }^{91}$ Huon, laisse 66 ; Berners, p. 153.

${ }^{92}$ Huon, laisse 91 ; Berners, p. 261-3.

${ }^{93}$ II. $1.169 \& 173$.

${ }^{94}$ Voir II.2.35-42 ; III.2.102-9 ; IV.1.68-70.

${ }^{95}$ V.1.383-402.

${ }^{96}$ Sur cette miniaturisation des fées et farfadets, figures inversées du monde solaire, voir aussi Gilbert Durand, Structures anthropologiques de l'imaginaire, Paris, Dunod, 1984 (1969), p. 225 et suivantes. Je remercie le professeur Jean Pironon pour ce précieux rappel.

${ }^{97}$ Voir aussi l'ouvrage de Claude Lecouteux, Les nains et les elfes au Moyen-Âge, Paris, Imago, 1997 (1988), qui fait d'Aubéron une figure de référence essentielle à l'analyse ethno-littéraire du nain.

${ }^{98}$ London, Malone Society, 1921. 99

I tell thee Bohan, Oberon is king

Of quiet, pleasure, profit and content,

Of wealth, of honor, and of all the world,

Tide to no place, yet all are tide to one,

Live thou in this life, exilde from world and men, 
And I will shew thee wonders ere we past

(638-643)

100

The Works of Ben Jonson, 7 vol., London, D. Widwinter, 1756 ; vol. 5: Masques at Court. 